\title{
Automation of the rapid plasma reagin test using the Autotape System
}

\author{
J. R. BOOTH, P. J. L. SEQUEIRA, W. WAGSTAFF, I. HOPKINSON, D. GIBBS, \\ AND E. BENNETT \\ From the Sheffield Blood Transfusion Centre, Central Serological Laboratories, Manchester, and Vickers \\ Limited $^{1}$
}

SUMMARY The paper describes the Autotape System, a new piece of apparatus which is automated slide testing equipment, and its successful application to the rapid plasma reagin test.

Large numbers of routine and repetitive tests create an obvious need for automation. The Technicon continuous-flow system has been developed for both haemagglutination (Sturgeon et al., 1963; Marsh et al., 1968) and syphilis (Wagstaff et al., 1969) tests in blood transfusion centres. The Autotape System equipment, which performs automated agglutination tests, was first used for brucellosis testing in cattle (Gower et al., 1974), and the second prototype has been set up for syphilis testing (Hopkinson et al., 1974) using a modification of the rapid plasma reagin (RPR) test (Portnoy et al., 1957).

\section{Material and method}

\section{SAMPLES}

Clotted whole blood samples were obtained from blood donors, together with serum and cerebrospinal fluid samples supplied by the Central Serological Laboratories, Manchester. Lipaemic sera are acceptable, but sera with any precipitate cannot be used without prior centrifugation. A constant level insert is placed into a 'Vacutainer' tube containing the samples (Gower et al., 1974). The tops of these are sealed off either individually, using a gloved finger, or in racks of up to 50 , using $13 \mathrm{~mm}$ polyester foam coated with $0.025 \mathrm{~mm}$ polythene and mounted on a $12 \mathrm{~mm}$ block of wood. The tubes are then inverted and vigorously reverted to throw the sera into the insert. The tubes with filled inserts are then centrifuged at $500 \mathrm{~g}$ for 5 minutes.

${ }^{1}$ The project is now being continued by G. D. Searle and Company.

Received for publication 30 June 1976
RPR TEST REAGENT

Antigen

VDRL antigen supplied by

Wellcome Laboratories

Limited

Buffer

$\mathrm{Na}_{2} \mathrm{HPO}$ (Analar)

$\mathrm{KH}_{2} \mathrm{PO}_{4}$ (Analar)

$2 \cdot 84 \mathrm{~g}$

Merthiolate (Thiomersal)

$2 \cdot 00 \mathrm{~g}$

Distilled water to 1 litre

This buffer has a pH of 6.9

Choline chloride (BDH biochemical grade) $40.00 \mathrm{~g}$ Distilled water to $100 \mathrm{ml}$

Disodium EDTA $\mathrm{Na}_{2}$ EDTA

$9 \cdot 3 \mathrm{~g}$ solution

Distilled water to $80 \mathrm{ml}$

Adjust to $\mathrm{pH} 7.0$ with $\mathrm{N}$

$\mathrm{NaOH}$ and make final

volume to $100 \mathrm{ml}$

\section{Preparation of the reagent}

To $0.4 \mathrm{ml}$ buffer in a flat-bottomed, stoppered, $30 \mathrm{ml}$ flask add $0.5 \mathrm{ml}$ VDRL antigen with continuous rotation of the flask. The antigen is added over the space of 5-6 seconds and another $2 \cdot 1 \mathrm{ml}$ of buffer is added. To this, add $1.25 \mathrm{ml}$ of choline chloride, 0.25 $\mathrm{ml}$ EDTA, and $0.5 \mathrm{ml}$ distilled water. Some batches of reagent then require vigorous shaking.

The reagent is made up fresh each day and is suitable for testing serum or plasma without inactivation.

\section{Autotape System}

The Autotape System (AS) is an automatic slide test apparatus (Figs. 1 and 2), in which tests are carried out on a continuous and constantly moving $35 \mathrm{~mm}$ transparent, sprocketed tape. The tape, which has been previously coated to give it suitable surface 


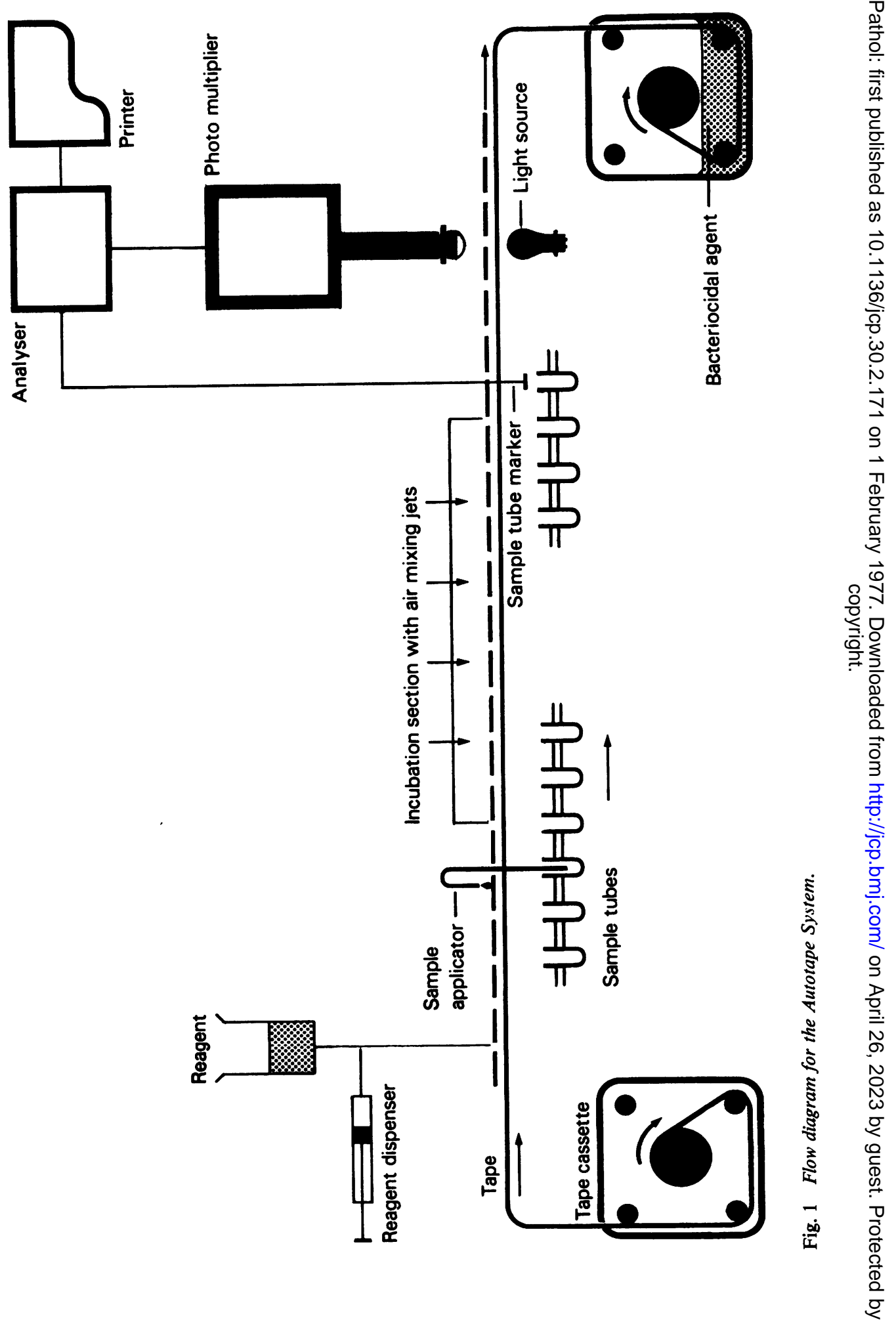




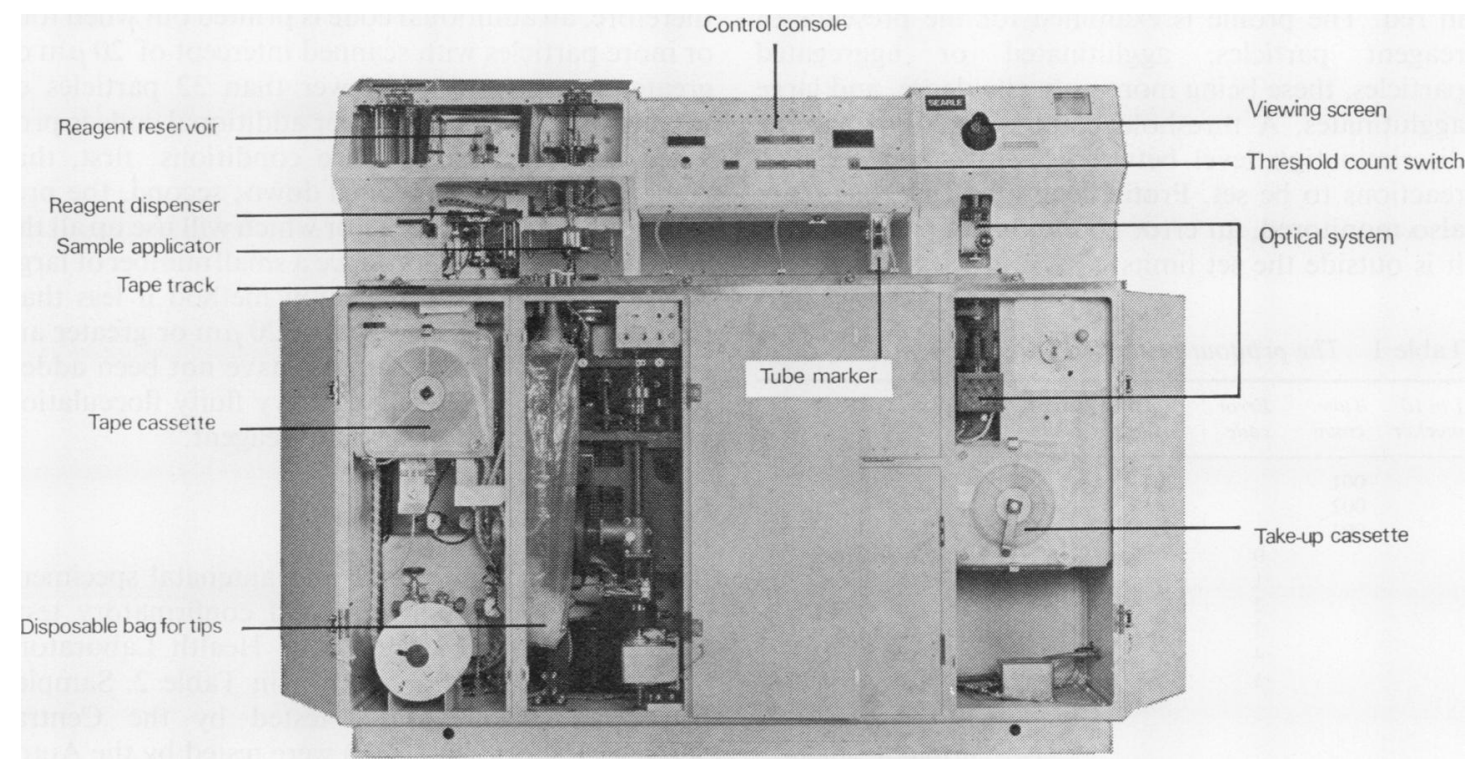

Fig. 2 The Autotape System.

tension properties, is driven from a cassette at the left-hand side of the apparatus by a constant-speed motor and taken up into a cassette at the right-hand side using a slipping clutch device run from the same motor. The speed of the tape can be set at one of two speeds, these settings being variable to provide incubation times of between 3 and 7 minutes.

The reagents are stored in a refrigerated compartment and are put on the tape by pneumatically driven pens, the predetermined volume being metered by Hamilton syringes, also pneumatically driven. When the pen is in the higher position, the syringes are activated so that an aliquot of reagent is drawn from the reservoir; at the same time a rocker bar mechanism seals the reagent dispense line between the syringe and the pen. In the lower position the pen is in contact with the tape, the rocker bar seals the tube between the syringe and reservoir, releasing its previous position, and the reagent is placed on the tape by depression of the syringe plunger. The length of time the pen is in the down position depends on the speed of the tape, and is set to give a profile of reagent of $12 \times 4 \mathrm{~mm}$, there being $8 \mathrm{~mm}$ between each profile.

The next section is the sample head. This consists of four collettes equally spaced in a circular metal disc of $200 \mathrm{~mm}$ diameter, which allows a separate tip to be used for each sample and test. Nylon tubing is fed from a roll into the tube feed mechanism where it is straightened by heat, moved up by $40 \mathrm{~mm}$ lengths using pneumatic clamps, and cut into $\mathbf{4 0} \mathrm{mm}$ lengths by a continuously revolving blade. The collettes are lowered and, as they rise again, a tip is fed into the collette above the tube feed mechanism, an airtight seal being formed by adjustable silicone $O$ rings. The collette then turns to the second station, where, when the head is lowered, the nylon tip enters the serum in the constant level insert and an aliquot is removed by a pneumatically operated preset Hamilton syringe. It then turns to the third station where in the lower position the aliquot of serum is added to the reagent profile. At the fourth station the tip is removed by automatically operated calipers and dropped into a disposable bag, leaving the collette ready for a new tip.

The sampling head is followed by a mixing and incubation chamber which is a covered length of 800 $\mathrm{mm}$. Circulating water under the track gives temperature control, and jets of humidified air, incorporated in the cover, provide the mixing. The profiles then pass over an edge detector, which detects the leading and trailing edges, and under an optical microscope which projects an image of the sample onto a viewing screen. By means of a beamsplitting device a $7 \mu \mathrm{m}$ wide scan is also projected onto a photo multiplier. The resulting output is a signal containing a series of voltage changes whose frequency and strength depend on the degree of agglutination in the test specimen, this output being processed by an electronic analyser. The processed signal results in a printout of six columns (Table 1), negatives in black and positives and error conditions 
in red. The profile is examined for the presence of reagent particles, agglutinated or aggregated particles, these being more optically dense, and large agglutinates. A threshold count switch enables the discrimination level between positive and negative reactions to be set. Profile length and thickness are also monitored, an error coding being printed out if it is outside the set limits.

Table 1 The printout of the Autotape System

\begin{tabular}{|c|c|c|c|c|c|}
\hline $\begin{array}{l}1 \text { in } 10 \\
\text { marker }\end{array}$ & $\begin{array}{l}\text { Tube } \\
\text { count }\end{array}$ & $\begin{array}{l}\text { Error } \\
\text { code }\end{array}$ & $\begin{array}{l}\text { Particle } \\
\text { count }\end{array}$ & $\begin{array}{l}\text { Additional } \\
\text { coding }\end{array}$ & \\
\hline & $\begin{array}{l}001 \\
002 \\
003\end{array}$ & $\begin{array}{l}\cdot 0 \\
\cdot 1 \\
\cdot 2 \\
\cdot 3 \\
\cdot 4 \\
\cdot 5\end{array}$ & $\begin{array}{l}004- \\
045+ \\
008-\end{array}$ & $\begin{array}{l}\mathbf{N} \\
\mathbf{P} \\
\mathbf{P} \\
\mathbf{P} \\
\mathbf{P} \\
\mathbf{P} \\
4 \\
\mathrm{~g} \\
\mathrm{P} \\
1 \\
\mathrm{C}\end{array}$ & $\begin{array}{l}\text { No tube in carrier } \\
\text { Profile too thick } \\
\text { Profile too long } \\
\text { Profile long and thick } \\
\text { Profile too short } \\
\text { Profile short and thick } \\
4 \text { or more particles } \\
\text { greater than } 20 \mu \mathrm{m} \\
\text { No reagent particles } \\
1 \text { and } 2 \text { on additional } \\
\text { coding }\end{array}$ \\
\hline
\end{tabular}

Running parallel with and mechanically interlocked to the tape is the sample conveyor system, each carrying 200 tubes at one loading. These tubes are identified by sequential numbering on the printout, and every tenth tube is sequentially identified by a 1 in 10 marker. To start the printout mechanism a tube must be present in the conveyor and at the same time a profile must be present on the tape. Subsequently, in the absence of a sample tube, the printout records a 'no tube in carrier' condition. A marker is activated by a positive or error condition, selecting those samples in the conveyor which correspond with the profile producing such conditions. Identification is thus automatic and precise.

The RPR test adds $7 \cdot 5 \mu \mathrm{l}$ of serum to $2.5 \mu \mathrm{l}$ of reagent, the mixture being incubated at $29^{\circ} \mathrm{C}$ and mixed at a flow rate of $41 / \mathrm{min}$ and a $\mathrm{RH}$ of $55 \%$ for $5 \mathrm{~min}$, giving a sample rate of 480 per hour.

To discriminate between positive reactions, negative reactions, and 'no serum added', settings on the analyser were adjusted using a series of known reagents and sera. A sample is classed as negative when a combination of the following two criteria has been fulfilled: 32 or more particles with the scanned intercept of $>7 \mu \mathrm{m}$ and with an optical density above a predetermined level, these being the reagent particles: and less than 15 particles with a scanned intercept of $>7 \mu \mathrm{m}$ at a different and higher optical density. A sample with 15 or more of the latter particles is classed as positive. A strong positive may have less than 15 particles available to be scanned; therefore, an additional code is printed out when four or more particles with scanned intercept of $20 \mu \mathrm{m}$ or $\overrightarrow{\vec{\sigma}}$ greater are scanned. If fewer than 32 particles of reagent are detected, a further additional code is pro- $\overrightarrow{\bar{s}}$ vided, implying one of three conditions: first, that $\overline{0}$ reagent has not been penned down; second, the pre- $\frac{}{O}$ sence of a very strong reactor which will use up all the $\overline{\bar{p}}$ available reagent and produce a small number of large $\Phi$ particles, providing a detection method if less than four particles with intercepts of $20 \mu \mathrm{m}$ or greater are scanned; third, serum samples have not been added $\vec{\circ}$ to the profile, producing a heavy fluffy flocculation $\overrightarrow{\vec{H}}$ which leaves little background reagent.

\section{Results}

A series of random donor and antenatal specimens were tested on this system and confirmatory tests were carried out by the Public Health Laboratory 0 Service. The results are shown in Table 2. Samples from patients previously tested by the Central 7 Serology Laboratory (CSL) were tested by the Auto- 음 tapeSystem(Table 3). These samples had been selected by the CSL for confirmatory testing from about 50000 specimens received by the laboratory and included $\vec{\theta}$ specimens referred to the Reference Laboratogy $\exists$ from outside the region and specimens for routio testing. Where technically possible all specimess were examined using the cardiolipin Wasserman reaction (CWR), the Reiter protein complement fixation test (RPCF), the treponemal haemaggluti- $\frac{}{\circ}$ nation test (THA), (Sequeira and Eldridge, 1973), and, in the majority of specimens, the fluorescence $\overline{\vec{B}}$ treponemal antibody (absorbed) test (FTA (Abs)). 3 By collation of the clinical data on the request forms, the laboratory records starting from 1962, and the CSL laboratory result confirmed in a proportion of patients by direct communication with the clinician:submitting the samples, patients were classified into the following groups: untreated early syphilis, $\stackrel{\circ}{\circ}$ untreated syphilis of other stages, treated early syphilis, treated syphilis of other stages, biologicalo false positive (BFP) reactors, and normal individuals. There was, in addition, a small group of unclassified

Table 2 Results of the random donor and antenatal specimens tested

\begin{tabular}{lrllll}
\hline & & $\begin{array}{l}\text { No sera detected } \\
\text { as error }\end{array}$ & Negative & Positive \\
\hline $\begin{array}{l}\text { Sera tested } \\
\text { No sera available } \\
\text { Total }\end{array}$ & $\begin{array}{r}15871 \\
421\end{array}$ & 421 & 15850 & $21^{1}$ \\
\hline
\end{tabular}

$\begin{array}{lr}\text { 1Positive sera. } & 17 \\ \text { False positive } & 3 \\ \text { Probable Treponemal infection } & 1 \\ \text { Biological false positive } & 1\end{array}$ 
Table 3 Comparison of the Autotape System results with the Central Serological Laboratory results

\begin{tabular}{|c|c|c|c|c|c|c|c|c|c|c|}
\hline & & \multicolumn{2}{|c|}{ Untreated syphilis } & \multicolumn{2}{|c|}{ Treated syphilis } & \multirow[t]{2}{*}{$B F P$} & \multirow[t]{2}{*}{ Normal } & \multirow[t]{2}{*}{ Unclassified } & \multirow[t]{2}{*}{ Total } & \\
\hline & & Early & Other & Early & Other & & & & & \\
\hline CWR & \pm & $\begin{array}{r}12 \\
2\end{array}$ & $\begin{array}{l}43 \\
10\end{array}$ & $\begin{array}{l}18 \\
21\end{array}$ & $\begin{array}{l}42 \\
71\end{array}$ & $\begin{array}{r}61 \\
3\end{array}$ & $\begin{array}{r}0 \\
122\end{array}$ & $\begin{array}{r}6 \\
26\end{array}$ & $\begin{array}{l}182 \\
253\end{array}$ & \\
\hline RPCF & \pm & $\begin{array}{r}13 \\
1\end{array}$ & $\begin{array}{r}45 \\
7\end{array}$ & $\begin{array}{l}15 \\
24\end{array}$ & $\begin{array}{l}55 \\
58\end{array}$ & $\begin{array}{r}0 \\
64\end{array}$ & $\begin{array}{r}0 \\
122\end{array}$ & $\begin{array}{r}8 \\
24\end{array}$ & $\begin{array}{l}136 \\
300\end{array}$ & \\
\hline THA & + & $\begin{array}{r}10 \\
4\end{array}$ & $\begin{array}{r}51 \\
0\end{array}$ & $\begin{array}{r}39 \\
0\end{array}$ & $\begin{array}{r}111 \\
2\end{array}$ & $\begin{array}{r}0 \\
62\end{array}$ & $\begin{array}{r}0 \\
107\end{array}$ & $\begin{array}{r}22 \\
2\end{array}$ & $\begin{array}{l}233 \\
177\end{array}$ & \\
\hline FTA (Abs) & + & $\begin{array}{r}12 \\
2\end{array}$ & $\begin{array}{r}49 \\
0\end{array}$ & $\begin{array}{r}25 \\
6\end{array}$ & $\begin{array}{r}92 \\
3\end{array}$ & $\begin{array}{r}0 \\
62\end{array}$ & $\begin{array}{r}0 \\
107\end{array}$ & $\begin{array}{r}7 \\
23\end{array}$ & $\begin{array}{l}185 \\
203\end{array}$ & 00 \\
\hline AS & $\stackrel{+}{-}$ & $\begin{array}{r}12 \\
2\end{array}$ & $\begin{array}{r}52 \\
1\end{array}$ & $\begin{array}{l}14 \\
25\end{array}$ & $\begin{array}{l}74 \\
39\end{array}$ & $\begin{array}{r}8 \\
56\end{array}$ & $\begin{array}{r}0 \\
122\end{array}$ & $\begin{array}{r}9 \\
23\end{array}$ & $\begin{array}{l}169 \\
268\end{array}$ & \\
\hline Total & & 14 & 53 & 39 & 113 & 64 & 122 & 32 & 437 & \\
\hline
\end{tabular}

patients. 'Early syphilis' included patients with primary, secondary, and latent syphilis in the first year of infection. 'Other stages' included latent and late symptomatic syphilis, a small number of patients with yaws and late congenital syphilis, and probably a small number of patients with early syphilis who had been incorrectly classified. The group of 'untreated' patients might be expected to include a small proportion of treated patients in whom no record of treatment had been found or had been treated inadvertently during the course of another illness. The BFP reactors were those patients whose sera gave positive reactions with either or both the CWR or AS tests. The normal group gave negative reactions with all tests used but were retested because of either trace reactions on the original testing,'clinical findings, or the specimen had been sent for confirmatory testing by other laboratories. The small remaining group of unclassified patients probably included a high proportion with treated primary syphilis. Table 3 shows the results obtained with the five tests: + includes strongly and weakly reactive results and - no reactive results. FTA (Abs) tests were not carried out on all samples. To simplify the table, non-valid results are omitted. It will be seen that, excluding the THA test, there is no significant difference between the sensitivities of the other four tests in untreated early syphilis. This group included one patient in whom Treponema pallida were demonstrated by dark-ground examination in a primary lesion, but all five tests gave negative results. In the other untreated group, the sensitivity of the AS test was not significantly less than the THA and the FTA (Abs) test, but very significantly greater than that of the RPCF and CWR tests. Thus, in the untreated group, the AS test was as sensitive as any of the four other tests used. In treated early syphilis, the AS test, the CWR, and RPCF tests were of virtually identical sensitivity but very significantly less sensitive than the 'long memory' THA and FTA (Abs) tests. In patients with treated syphilis of other stages, the sensitivity of the AS test is very significantly greater than the CWR and RPCF tests but very significantly less than the THA and FTA (Abs) tests. Among the $61 \mathrm{BFP}$ reactions with the CWR, only five gave positive reactions with the AS test. However, among 125 'normal' specimens, three gave false positive reactions with the AS test and not with the CWR.

\section{Discussion}

The printout from the analysed results gives an easyto-read set of figures, those in black indicating a negative result, and those in red requiring further tests to differentiate between a positive and an error condition. As a screening test it has a very high rate of detection of untreated syphilis, and a very high specificity demonstrated by its performance in the donor material and in the BFP sera from the CSL. The detection rate in untreated syphilis was superior to any single screen test at the CSL. The development of a second test using the AS based on the THA test should provide a test system of very high standard for the detection and control of syphilis.

Discrete testing on a moving tape and the use of a fresh tip for each sample eliminates all carry-over. Some batches of reagent give weak false positive reactions; these are the batches which require vigorous shaking before use.

Clean serum must be used to eliminate a false negative condition. A serum which is a very strong reactor produces very large particles, insufficient in number to be interpreted by the analyser on the additional coding. Normally, this would give an error condition of 'no reagent detected', but if there are 
particles of the appropriate size already present in the serum these will be interpreted as being reagent particles giving a false negative result. Lipaemic sera do not cause this error.

The AS system introduces into the laboratory direct automation of slide tests under standard and, therefore, reproducible conditions. In theory, this equipment can carry out and read any agglutination slide test once the volumetric and incubation time adjustments have been made and the decisive criteria for 'positive' or 'negative' have been set. The RPR test shows itself to be capable of successful application.

\section{References}

Gower, S. G. M., Wright, E. C., Davies, G., Brinley Morgan, W. J., Hopkinson, W. I., Gibbs, D. F., and Bennet, E. J. (1974). An automated Rose Bengal agglutination test using the ADAM system. Veterinary Record, 95, 544-547.

Hopkinson, W. I., Gibbs, D. F., and Bennet, E. J. (1974): A new concept in the automation of the VDRL test preliminary results. Antonie van Leeuwenhoek, 40, 609.

Marsh, W. L., Nichols, M., and Jenkins, W. J. (1968) Automated detection of blood group antibodies $\overline{\bar{n}}$ Journal of Medical Laboratory Technology, 25, 335-342.

Portnoy, J., Garson W., and Smith, C. A. (1957). Rapide plasma reagin test for syphilis. Public Health Reportsw (Washington), 72, 761-766.

Sequeira, P. J. L. and Eldridge, A. E. (1973). TreponemaP haemagglutination test. British Journal of Venerea $L$ Diseases, 49, 242-248.

Sturgeon, P., Cedergren, B., and McQuiston, D. (1963)증 Automation of routine blood typing procedures. Vox Sanguinis, 8, 438-451.

Wagstaff, W., Firth, R., Booth, J. R., and Bowley, C. Cị (1969). Large-scale screening by the automated Wassermann reaction. Journal of Clinical Pathology, 22, 236-239. 